\title{
Partial order in Potts models on the generalized decorated square lattice
}

\author{
M. P. Qin, ${ }^{1}$ J. Chen, ${ }^{1}$ Q. N. Chen,${ }^{2}$ Z. Y. Xie,${ }^{1}$ X. Kong, ${ }^{1}$ H. H. Zhao, ${ }^{1}$ B. Normand,${ }^{3}$ and T. Xiang ${ }^{1}$ \\ ${ }^{1}$ Institute of Physics, Chinese Academy of Sciences, Beijing 100190, China \\ ${ }^{2}$ Department of Chemistry, Frick Laboratory, Princeton University, Princeton, New Jersey 08544, USA \\ ${ }^{3}$ Department of Physics, Renmin University of China, Beijing 100872, China
}

(Dated: March 13, 2021)

\begin{abstract}
We explore the Potts model on the generalized decorated square lattice, with both nearest $\left(J_{1}\right)$ and next-neighbor $\left(J_{2}\right)$ interactions. Using the tensor renormalization-group method augmented by higher-order singular value decompositions, we calculate the spontaneous magnetization of the Potts model with $q=2,3$, and 4 . The results for $q=2$ allow us to benchmark our numerics using the exact solution. For $q=3$, we find a highly degenerate ground state with partial order on a single sublattice, but with vanishing entropy per site, and we obtain the phase diagram as a function of the ratio $J_{2} / J_{1}$. There is no finite-temperature transition for the $q=4$ case when $J_{1}=J_{2}$, but the magnetic susceptibility diverges as the temperature goes to zero, showing that the model is critical at $T=0$.
\end{abstract}

PACS numbers: 64.60.Cn, 05.50.+q, 75.10.Hk, 64.60.F-

The $q$-state Potts model plays an important role in the understanding of different phases and critical phenomena (for a review see Ref. [4]). The behavior of ferromagnetic Potts models is well understood due to their universality. The properties of antiferromagnetic Potts models are much more complex, varying widely for different values of $q$ and lattice topologies. On every lattice there exists a value $q_{c}$ such that for $q>q_{c}$ the model has exponentially decaying correlations and hence no order at all temperatures, including $T=0$. For $q=q_{c}$, the model has a critical point at zero temperature, and for $q<q_{c}$ all types of behavior are possible [2], including long-ranged order or partial order. When the lattice is irregular, meaning that not all sites are equivalent, entropy-driven phase transtions and exotic forms of partial order may arise 3 5. Partial order has been found both in classical statistical models [6, 7] and in quantum systems [8].

The decorated square lattice is formed by adding one site to each bond of a square lattice. Here we study Potts models on the generalized decorated square lattice obtained by introducing an interaction between the decorating sites [Fig. [1(a)]. This is a tripartite lattice, for which one attribution of sublattices $(A, B, C)$ is shown in Fig. 1(b). The Hamiltonian of the model is

$$
H=J_{1} \sum_{\langle i, j\rangle} \delta_{\sigma_{i}, \sigma_{j}}+J_{2} \sum_{\langle\langle i, j\rangle\rangle} \delta_{\sigma_{i}, \sigma_{j}}-h \sum_{i \in \mathcal{L}} \delta_{\sigma_{i}, 0},
$$

where $\sigma_{i}=0,1,2, \ldots$ is the Potts state on lattice site $i$. The first term denotes all nearest-neighbor bonds and the second all next-neighbor bonds in Fig. 11(a), while the third term denotes a field term $h$ applied to the sites in any sublattice(s) $\mathcal{L}$ for the calculation of magnetizations and susceptibilities.

In the pure decorated square lattice, with only nearestneighbor $\left(J_{1}\right)$ interactions, taking a partial trace over the decorating ( $B$ and $C$ ) sites effects a mapping to a ferromagnetic Potts model on the square lattice 911 . From

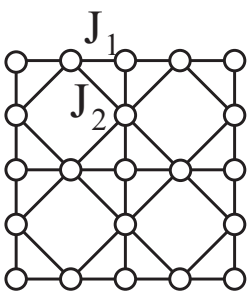

(a)

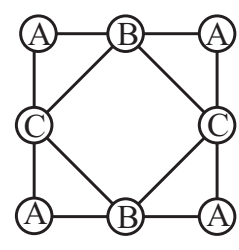

(b)

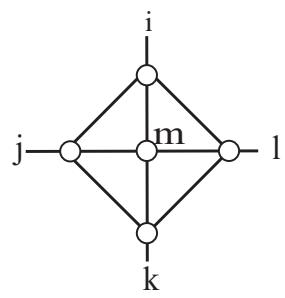

(c)
FIG. 1: (a) Generalized decorated square lattice with interactions between decorating sites. (b) Sublattice labels for this tripartite lattice. (c) Label definition for the tensor expressed in Eq. (2).

this one may deduce that the critical value for the $q$-state antiferromagnetic Potts model on the decorated square lattice is $q_{c}=\frac{1}{2}(3+\sqrt{5})=2.618$, meaning that for $q=3$ and $q=4$ there is no ordered phase at low temperatures and no phase transition occurs. This value of $q_{c}$ is smaller than that for the antiferromagnetic Potts model on the square lattice, where $q_{c}=3 \sqrt{12}$ 16. For $q=2$ (the Ising model), the exact solution is one with complete long-range order [17].

When next-neighbor interactions are introduced, reentrant behavior of the ordered phases has been found both for $q=2$ [17, 18] and $q=3$ [19]. However, this phenomenon required that one of the interactions be ferromagnetic [19]. Here we consider the regime where both $J_{1}$ and $J_{2}$ are antiferromagnetic and focus primarily on the isotropic situation $\left(J_{1}=J_{2}\right)$, where the competition between the different interactions is the largest.

For a classical model on any lattice, it is always possible to find a tensor-network representation of the partition function [20, 21. For the Hamiltonian defined in Eq. (1), we define a rank-four tensor $T_{i j k l}$ for the unit 
cell shown in Fig. 11(c) as

$$
\begin{array}{r}
T_{i j k l}=\sum_{m} \exp \left[-\beta J_{1}\left(\delta_{\sigma_{i}, \sigma_{m}}+\delta_{\sigma_{j}, \sigma_{m}}+\delta_{\sigma_{k}, \sigma_{m}}+\delta_{\sigma_{l}, \sigma_{m}}\right)\right] \times \\
\exp \left[-\beta J_{2}\left(\delta_{\sigma_{i}, \sigma_{j}}+\delta_{\sigma_{j}, \sigma_{k}}+\delta_{\sigma_{k}, \sigma_{l}}+\delta_{\sigma_{l}, \sigma_{i}}\right)\right] . \quad(2)
\end{array}
$$

The Potts variables $\sigma_{i}$ on each site serve as the indices of the tensors, and the tensors for each unit cell form a square-lattice tensor network.

Recently, we have developed a Tensor Renormalization Group (TRG) technique which makes use of higherorder singular value decomposition (HOSVD) of the tensors to truncate them during the renormalization (coarsegraining) process [23]. There are two steps in this method at each iteration $n$. In the first, we block two tensors to form a larger tensor,

$$
M_{x x^{\prime} y y^{\prime}}^{(n)}=\sum_{i} T_{x_{1} x_{1}^{\prime} y i}^{(n)} T_{x_{2} x_{2}^{\prime} i y^{\prime}}^{(n)}
$$

where $x=x_{1} \otimes x_{2}$ and $x^{\prime}=x_{1}^{\prime} \otimes x_{2}^{\prime}$. In the second, we perform a HOSVD,

$$
M_{x x^{\prime} y y^{\prime}}^{(n)}=\sum_{i j k l} S_{i j k l} U_{x i}^{L} U_{x^{\prime} j}^{R} U_{y k}^{U} U_{y^{\prime} l}^{D}
$$

where the $U$ matrices are unitary and $S$ is the core tensor of $M^{(n)}$, which is fully orthogonal and pseudo-diagonal 23. The tensor is then truncated according to

$$
T_{x x^{\prime} y y^{\prime}}^{(n+1)}=\sum_{i j} U_{i x}^{(n+1)} M_{i j y y^{\prime}}^{(n)} U_{j x^{\prime}}^{(n+1)}
$$

to obtain a new tensor $T^{(n+1)}$ with the same dimension as $T^{(n)}$. In one "HOTRG" step, the size of the lattice is reduced by a factor of $1 / 2$. By repeating the HOTRG step alternately in the $x$ and $y$ directions, we contract the tensor network and obtain the partition function of the model.

While several methods exist to contract the tensor network [20, 21, 25, 26], we have demonstrated [23] that the accuracy of this HOTRG method is very high: a tensor dimension of just $D=24$ in the two-dimensional Ising model is sufficient to ensure that the relative error in the free energy is less than $10^{-7}$, even at the critical temperature. The accuracy can be further improved by including the effects of the environment in the renormalization process, known as the second renormalization group (SRG) method [24], although we do not need to implement this for the accuracies attained here.

For numerical reasons, the thermodynamic quantities obtained most accurately from the HOTRG partition function are the magnetization and susceptibility. The small field term included in Eq. (1) pins a state of $q=0$ order to a specific sublattice $\mathcal{L}$. For a starting square lattice of dimensions $L \times L=N$, we define the order parameter as

$$
M=\frac{1}{N}\left\langle\sum_{i \in \mathcal{L}} \delta_{\sigma_{i}, 0}\right\rangle-\frac{1}{q}
$$

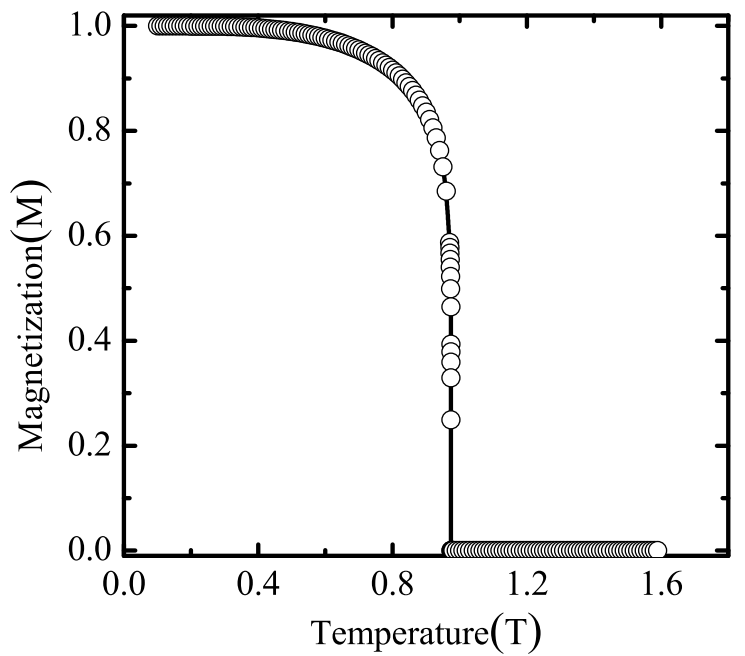

FIG. 2: Magnetization of the $q=2$ Potts model on the generalized decorated square lattice with $J_{1}=J_{2}=1$. The bond dimension in the HOTRG calculation is $D=30$. An Ising-like phase transition occurs at $T_{c}=0.97423$

which is the expectation value of the number of spins in sublattice $\mathcal{L}$ whose value is 0 . The term $1 / q$ is subtracted to ensure a value of 0 in the disordered phase. The susceptibility is given by $\chi=\partial M / \partial h$. The order parameter $M$ expresses the broken sublattice symmetry 22] of the partially ordered phase: only sites of one sublattice are ordered, while all others are disordered (but may still be correlated locally). An ordered state with broken sublattice symmetry was first suggested for the $q=3$ antiferromagnetic Potts model on the square lattice, but more detailed analysis demonstrated that this order is artificial, the model being disordered at all temperatures other than a $T_{c}=0$ critical point 12 15].

The generalized decorated square lattice model is exactly solvable 18 for $q=2$. There are three sublattices but only two possible states for each site, leading to frustration. Because the coordination number of the $A$ sites, $z_{A}=4$, is less than that of the $B$ and $C$ sites $\left(z_{B}=z_{C}=6\right)$, the ground state is one with Néel order on the $B$ and $C$ sublattices while the Ising spins on the $A$ sites are free. This configuration minimizes the number of unsatisfied bonds and hence the energy. The free spins are responsible for the zero-temperature residual entropy, $S_{0}=\frac{1}{3} \ln 2$ per site. The universality class of the phase transition from the Néel phase to the paramagnetic phase remains that of the two-dimensional Ising model. In Fig. 2 we show the magnetization obtained for $J_{1}=J_{2}$ with tensor dimension $D=30$ in the HOTRG calculation. The transition temperature we compute is $T_{c}=0.97423$, which is very close to the exact result $T_{c}=0.9742197$, demonstrating clearly the accuracy of the HOTRG method. 


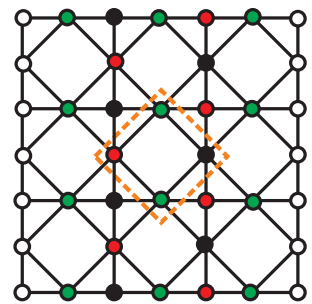

(a)

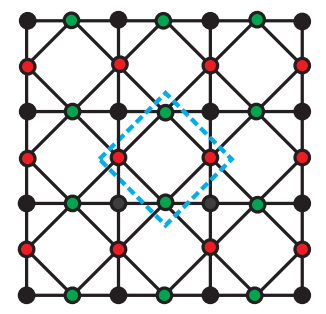

(c)

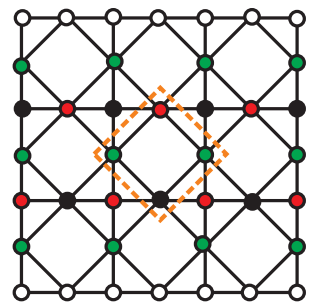

(b)

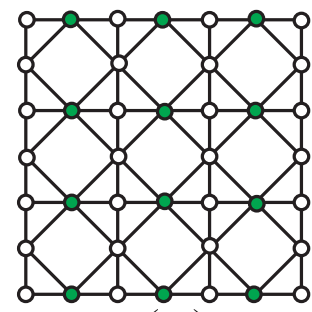

(d)
FIG. 3: Ground-state configurations for the $q=3$ Potts model on the generalized decorated square lattice. The three states are represented by red for 0 , black for 1 , and green for 2 . Panels (a) and (b) illustrate an arbitrarily chosen square on the $B$ and $C$ sublattices with one pair of diagonally opposite sites in the same state, which creates a complete partial order on the corresponding sublattice. In panel (c), both opposite pairs have the same state and all three sublattice are ordered. All of the ground-state configurations are summarized in panel (d) and a $\pi / 2$ rotation of this.

We turn now to the $q=3$ model and begin by developing a heuristic understanding of the underlying physics. Because each site may take one of three states and the lattice is tripartite, all bonds can be satisfied and this model is unfrustrated. If the three states are represented by three different colors, as in Fig. 3 , the problem of finding all the ground-state configurations may be cast as a coloring problem. In fact the division of sites into three sublattices shown in Fig. 1 (b) is not unique (a situation that would ensure the same type of order as in the $q=3$ Potts model on the triangular lattice). The origin of the different possible sublattice designations is the fact that $z_{A}=4$ for one of the sublattices, introducing more degrees of freedom and causing a degenerate ground state.

For the square of $B$ - and $C$-sublattice sites marked by the orange dashed line in Figs. B(a) and (b), at least one pair of diagonally opposite sites must have the same color. Each such square effectively nucleates a state of complete order on the relevant sublattice, as shown in Figs. 3(a) and (b), without ordering the other two sublattices (each of which in fact forms a state of two-fold degenerate alternating lines). If both pairs of opposite sites have the same color [Fig. 目(c)], then the nucleated state would be one of complete order on all three sublattices, but this is a set of measure zero. The set of all ground-

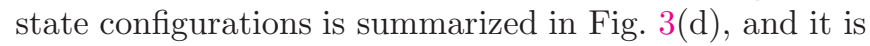

one with partial order on a single sublattice. Thus the additional freedom contained in the special topology of the generalized decorated square lattice is not sufficient to drive the system into a disordered phase.

To investigate the nature of the partially ordered state, if the $B$ sublattice is ordered with $q=0$, the state of the intervening lines of $A$ and $C$ sites may be either $1212 \ldots 12$ or $2121 \ldots 21$ at random, and similarly for an ordered $C$ sublattice. Thus the partially ordered state breaks the $\pi / 2$ rotation symmetry of the lattice. The ground-state degeneracy at zero temperature is $N_{0}=6\left(2^{L}-1\right)$, where 6 is subtracted because configurations in which both $B$ and $C$ sublattices are ordered [Fig. $3(c)]$ are double-counted. Despite this high degneracy, the residual entropy per site,

$$
S_{0}=\lim _{L \rightarrow \infty} \frac{\log \left(6 \times 2^{L}-6\right)}{L^{2}}=0,
$$

vanishes because of the one-dimensional nature of the freedom in ground-state configurations within a twodimensional lattice. This type of partial order is different from the order found in Refs. [1, 22], where only one sublattice was ordered and the other sites remained in random states. For the generalized decorated square lattice, the sites on lines between the ordered sublattice sites remain highly correlated, forming a one-dimensional antiferromagnetic Ising model $(q=2)$, which has twofold degeneracy between the alternating states as noted above.

We calculate the magnetization and susceptibility for the $q=3$ Potts model on the generalized decorated square lattice for $J_{1}=J_{2}$ using the HOTRG method with $D=30$. As shown in Fig. 1 , we find that the magnetization goes continuously to zero at a second-order phase transition at $T_{c}=0.528(1)$. At low temperatures, the magnetization defined by Eq. (6) with $\mathcal{L} \equiv C$ takes the value $2 / 3$, which means that all $C$ sites are fully polarized and confirms our analysis concerning the nature of the ground state. The susceptibility (inset, Fig. (1) shows a clear divergence at $T_{c}$, confirming the secondorder nature of the phase transition.

Having established the physics of partial order where $J_{1}=J_{2}$, we next investigate the regime where the bonds are not unequal $\left(J_{1} \neq J_{2}\right)$. We consider only the antiferromagnetic region where $J_{1}, J_{2}>0$. In the limit where $J_{2}=0$, the system is a pure decorated square lattice with $q_{c}=2.618$ and a disordered ground state for $q=3$. In the limit where $J_{1}=0$, the system is a $q=3$ Potts model on the square lattice formed by the decorating $B$ and $C$ sites, while the $A$ sites become isolated. For the antiferromagnetic Potts model on the square lattice, the critical value is $q_{c}=3$ [16], and hence $T_{c}=0$ here. The phase diagram we calculate is presented in Fig. . with the critical temperature shown in units of $J_{1}$ when $J_{1}>J_{2}$ and $J_{2}$ when $J_{2}>J_{1}$. 


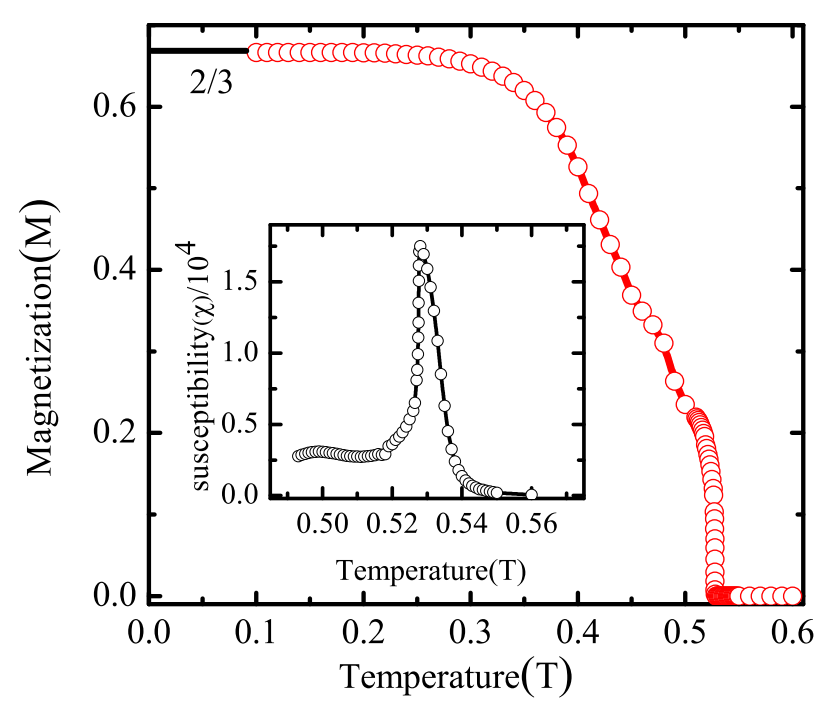

FIG. 4: Magnetization for the $q=3$ Potts model on the generalized decorated square lattice with $J_{1}=J_{2}$ and $D=30$. A second-order phase transition occurs at $T_{c}=0.528(1)$. The inset shows the susceptibility, which diverges at $T_{c}$.

Considering first the situation $J_{2}<J_{1}$, we find a finitetemperature phase transition to a state of partial order for all $J_{2}$, and that $T_{c} \rightarrow 0$ continuously as $J_{2} \rightarrow 0$. This implies that $q_{c} \geq 3$ in this limit. However, it is known that $q_{c}=\frac{1}{2}(3+\sqrt{5})=2.618$ at $J_{2}=0$ [9 11], meaning a disordered ground state at $T=0$, and hence we find discontinuous behavior here. The key property is a qualitative one, the connectivity of the lattice, and not a quantitative one related to the coupling ratio. This result may in fact be anticipated from the fact that the ground state should be the same in the whole region $J_{1}, J_{2}>0$, because it corresponds to the same coloring problem, and hence $T_{c}$ should be finite for all $J_{2}>0$.

We have not been able to find a similar example of such a discontinuity anywhere in the literature. We note in this context that the applied field acts to induce order at finite temperatures in the antiferromagnetic Ising model on the triangular lattice and the $q=3$ Potts model on square lattice [27, both of which are critical at $T=0$ in the absence of a field. In principle our method is susceptible to this type of physics, because our calculations use a small applied field, and so we have verified our re-

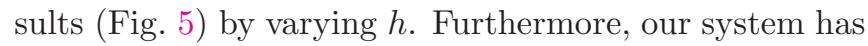
the essential difference that the ground state of the decorated square lattice is not critical but is fully disordered (exponentially decaying correlations) in the absence of a field. At the other side of the phase diagram in Fig. 5 , obtained by taking $J_{1} \rightarrow 0$, the model becomes critical at zero temperature and fluctuations become very large [14. In Fig. 目 we show the critical temperature calculated for moderate ratios $J_{2} / J_{1}$, and we appeal to known results in expecting that $T_{c}$ continues smoothly to zero

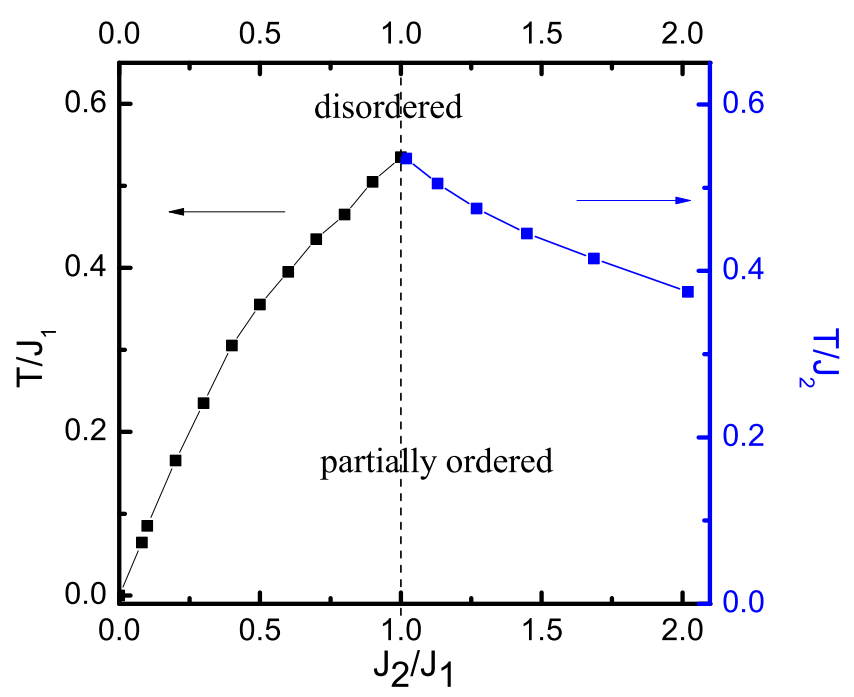

FIG. 5: Phase diagram for the $q=3$ Potts model on the generalized decorated square lattice as a function of $J_{2} / J_{1}$. HOTRG calculations for the critical temperature $T_{c}$ were performed with $D=30$. The temperature is expressed in units of $J_{1}$ for $J_{2} / J_{1}<1$ and in units of $J_{2}$ when $J_{2} / J_{1}>1$.

when $J_{1} \rightarrow 0$.

We conclude our analysis of the generalized decorated square lattice with the $q=4$ case, where we consider only $J_{2}=J_{1}$. For this value of $q$, the antiferromagnetic Potts model on the Union-Jack lattice was found to have a finite-temperature phase transition to a partially ordered ground state [4, 5. The generalized decorated square lattice with $J_{2}=J_{1}$ may be considered as the result of eliminating half of the fourfold-coordinated centering sites within the squares of the Union-Jack lattice. This weakens the constraints on the ground state, which thus has sufficient freedom that a disordered state may well be expected: there are four states per site and the lattice is tripartite, with many ways to divide the system into three sublattices.

We compute the magnetization by the HOTRG method, finding no order over the whole temperature range. However, when we calculate the susceptibility by adding a very small magnetic field term to the system $\left(h / J_{1}=10^{-6}\right)$, we find (Fig. 6) that it diverges in the limit of zero temperature. To analyze this divergence, in the inset of Fig. 同 we show the low-temperature behavior of $\chi(T)$ on logarithmic axes. The data show a robust scaling relation of the form

$$
\chi \sim T^{-\gamma},
$$

where the exponent $\gamma=1.0168(5)$. This scaling behavior indicates that the model is critical at zero temperature. Such zero-temperature critical behavior is also present in the antiferromagnetic $q=2$ (Ising) [28, 29] and $q=4$ Potts models on the triangular lattice [30], as well as the 


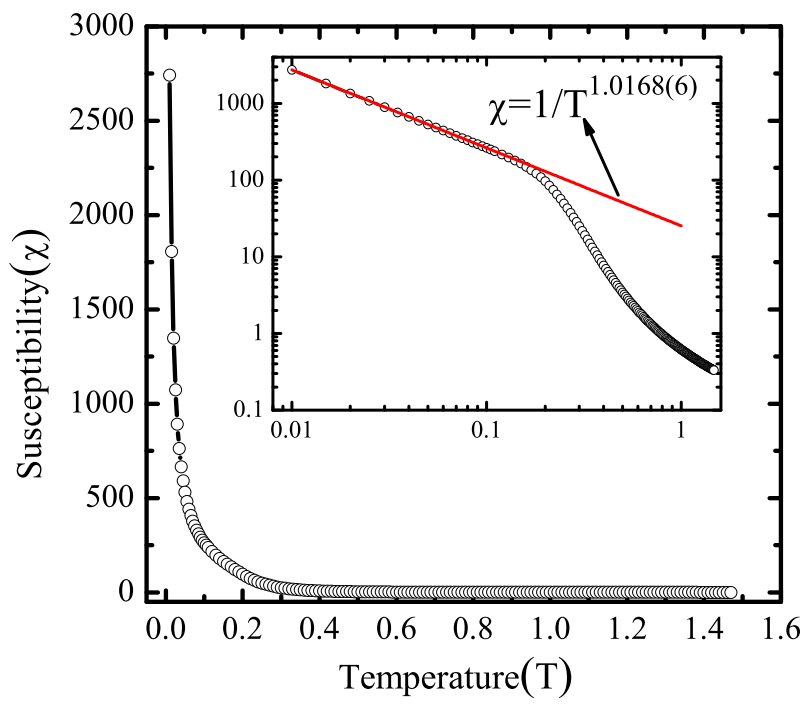

FIG. 6: Susceptibility for the $q=4$ Potts model on the generalized decorated square lattice with $J_{1}=J_{2}$ and $D=30$. The inset shows the behavior near $T=0$ on logarithmic axes, which displays good linear scaling with a slope of 1.0168(5).

$q=3$ model on the square lattice [16]. From our result we can infer that the critical point for the generalized decorated square lattice is not less than 4 , i.e. $q_{c} \geq 4$. In comparison with the triangular lattice, where $q_{c}=4$ [30] and the coordination $z=6$, the lattice we consider here has a lower average coordination number, $\bar{z}=16 / 3$, but is also irregular in the sense of having inequivalent sites, an effect now understood [-

To conclude, we study the antiferromagnetic Potts model on the generalized decorated square lattice by calculating its field-dependent thermodynamic properties using the HOTRG method. For $q=3$, we find a secondorder phase transition, occurring at $T_{c}=0.528(1)$ for the isotropic point $\left(J_{1}=J_{2}\right)$, to a state of partial order. The ground state has partial order on a single sublattice, with spontaneous breaking of $\pi / 2$ rotational symmetry and the permutation symmetry of the Potts model. The entropy of this highly degenerate state scales with the linear dimension of the system, meaning that the entropy per site vanishes at zero temperature. We obtain the phase diagram as a function of the ratio $J_{2} / J_{1}$, and our results indicate that a finite-temperature phase transition is always present, but that $T_{c} \rightarrow 0$ continuously as $J_{1} \rightarrow 0$ and discontinuously as $J_{2} \rightarrow 0$. Thus the physics is determined not by the ratio $J_{2} / J_{1}$ but by the lattice connectivity. The same model with $q=4$ is disordered at any finite temperature. However, for $J_{1}=J_{2}$ we find that the model is critical at zero temperature, the sus- ceptibility diverging with the form $\chi \sim T^{-1}$, and thus we infer that the critical value of $q$ in this lattice is $q_{c} \geq 4$.

Acknowledgements: this project was supported by the National Natural Science Foundation of China (Grant No. 10934008, 10874215 and 11174365), the National Basic Research Program of China (Grant No. 2012CB921704 and 2011CB309703).

[1] F. Y. Wu 1982 Rev. Mod. Phys. 54235

[2] J. Salas and A. D. Sokal 1997 J. Stat. Phys. 86551

[3] R. Kotecky, J. Salas, and A. D. Sokal 2008 Phys. Rev. Lett. 101030601

[4] Q. N. Chen, M. P. Qin, J. Chen, Z. C. Wei, H. H. Zhao, B. Normand, and T. Xiang 2011 Phys. Rev. Lett. 107 165701

[5] Y. J. Deng, Y. Huang, J. L. Jacobsen, J. Salas, and A. D. Sokal 2011 Phys. Rev. Lett. 107150601

[6] H. T. Diep and M. Debauche 1991 Phys. Rev. B 438759

[7] A. Lipowski and T. Horiguchi 1995 J. Phys. A 283371

[8] R. Quartu and H. T. Diep 1997 Phys. Rev. B 552975

[9] F. Y. Wu 1980 J. Stat. Phys. 23773

[10] Y. Matsuda, Y. Kasai, and I. Syozi 1981 Prog. Theor. Phys. 651091

[11] D. Hajdukovic 1983 J.Phys. A 162881

[12] M. P. Nightingale and M. Schick 1982 J. Phys. A 15 L39

[13] M. P. M. den Nijs, M. P. Nightingale, and M. Schick 1982 Phys. Rev. B 262490

[14] Z. Racz and T. Vicsek 1983 Phys. Rev. B 272992

[15] P. Reed 1985 J. Phys. C 18 L901

[16] S. J. Ferreira and A. D. Sokal 1999 J. Stat. Phys. 96461

[17] M. Debauche, H. T. Diep, P. Azaria, and H. Giacomini 1991 Phys. Rev. B 442369

[18] P. Azaria, H. T. Diep, and H. Giacomini 1987 Phys. Rev. Lett. 591629

[19] Y. Zhao, W. Li, B. Xi, Z. Zhang, X. Yan, S. J. Ran, T. Liu, and G. Su 2013 Phys. Rev. E 870.32151

[20] M. Levin and C. P. Nave 2007 Phys. Rev. Lett. 90120601

[21] H. H. Zhao, Z. Y. Xie, Q. N. Chen, Z. C. Wei, J. W. Cai, and T. Xiang 2010 Phys. Rev. B 81174411

[22] G. S. Grest and J. R. Banavar 1981 Phys. Rev. Lett. 46 1458

[23] Z. Y. Xie, J. Chen, M. P. Qin, J. W. Zhu, L. P. Yang, and T. Xiang 2012 Phys. Rev. B 86045139

[24] Z. Y. Xie, H. C. Jiang, Q. N. Chen, Z. Y. Weng, and T. Xiang 2009 Phys. Rev. Lett. 103160601

[25] G. Vidal 2007 Phys. Rev. Lett. 98070201

[26] G. Vidal 2007 Phys. Rev. B 78155117

[27] S. L. A. de Queiroz, T. Paiva, J. S. de Sa Martins, and R. R. dos Santos 1999 Phys. Rev. E 592772

[28] G. H. Wannier 1950 Phys. Rev 79357

[29] J. Stephenson 1970 J. Math. Phys. 11413

[30] C. Moore and M. E. J. Newman 2000 J. Stat. Phys. 99 629 